\title{
Simulação numérica de dois sistemas convectivos de mesoescala utilizando o modelo WRF
}

\author{
Numerical simulation of two mesoscale convective systems using the WRF model
}

\author{
Kauan Vargas Casarin \\ Universidade Federal de Santa Maria, Santa Maria, Brasil \\ kauancasarin@gmail.com
}

\begin{abstract}
Resumo
O modelo Weather Research and Forecasting (WRF) foi utilizado com o objetivo de simular dois Sistemas Convectivos de Mesoescala (SCMs) com diferentes características para analisar de que forma variáveis como cisalhamento direcional do vento e o gradiente de espessura modificam-se no interior dos SCMs ao longo de toda sua duração. O primeiro evento é um SCM linear que estendeu-se desde o noroeste da Argentina até o leste do Rio Grande do Sul em 30 de Novembro de 2009 e o segundo é um SCM circular ou CCM (complexo convectivo de mesoescala) que ocorreu sobre o RS e o Uruguai em 18 de Novembro de 2009. Os dois sistemas foram identificados através de imagens do satélite GOES (Geostationary Operational Environmental Satellite) usando um método de rastreamento automático de SCMs e para a simulação dos eventos no WRF foram utilizados os dados de reanálise do CFSR (Climate Forecast System Reanalysis). Os resultados da simulação indicaram que a taxa de redução do gradiente de espessura é maior no caso circular do que no caso linear e no momento de iniciação de ambos os eventos o cisalhamento direcional do vento é maior no caso linear, porém ele reduz até a dissipação do SCM diferente do que ocorre no CCM que apresenta um aumento do cisalhamento direcional do vento em um estágio próximo a dissipação do sistema.
\end{abstract}

Palavras-chave: SCM, mesoescala, WRF, cisalhamento, espessura.

\begin{abstract}
The model Weather Research and Forecasting (WRF) was used in order to simulate two Mesoscale Convective Systems (MCSs) with different characteristics in order to analyze how variables such as wind directional shear and thickness gradient are modified within the MCSs along its entire duration. The first event is a linear MCS that extended from the north of Argentina to the South Atlantic in November 30, 2009 and the second is a circular MCS or MCC (mesoscale convective complex) that occurred on the RS and Uruguay on November 18, 2009. The two systems were identified through images of the satellite GOES (Geostationary Operational Environmental Satellite) using an automatic tracking of MCSs and for the simulation of events in WRF we used the data reanalysis of CFSR (Climate Forecast System Reanalysis). The simulation results indicated that the rate of reduction thickness gradient is greater in the circular case than in the linear case and at the time that events are initiated the wind directional shear is higher in the linear case but he reduces until the moment of dissipation of the MCS different than occurs in MCC, which has an increased wind directional shear when the system is almost dissipating.
\end{abstract}

Keywords: MCS, mesoscale, WRF, shear, thickness. 


\section{Introdução}

Os sistemas convectivos de mesoescala (SCMs) são amplas tempestades que possuem grandes dimensões espaciais, longa duração e estão frequentemente associadas a altas taxas de precipitação, ventos fortes, trovoadas, granizo e eventualmente tornados. Os primeiros satélites ambientais colocados em orbita permitiram a identificação desses sistemas pois eles formam grandes escudos de nuvens que podem facilmente ser observados por imagens de satélite no canal infravermelho. Um trabalho realizado por Maddox (1980) determinou que um SCM deve possuir uma temperatura do topo de nuvens inferior a $-32^{\circ} \mathrm{C}$ em uma área continua de no mínimo $100.000 \mathrm{~km}^{2}$ durante um período maior que 6 horas.

Na América do Sul (AMS) esses sistemas convectivos ocorrem com maior frequência na região sul e sudeste (Bacia do prata e sul do Brasil) durante as estações quentes (primavera verão), isso ocorre devido a um fluxo maior e calor e umidade oriundo da região amazônica que se estabelece nessa época dono. Esse fluxo de norte, na maioria dos casos de SCMs, se manifesta na forma de um jato de baixos níveis (JBN). Um estudo realizado por Nicolini et al. (2002) evidencia a alta correlação entre os SCMs e o jato de baixos níveis, sendo que $81 \%$ de 27 SCMs estudados ocorreram associados ao JBN. Saulo et al. (2007) mostraram que $88,6 \%$ dos casos de verão e $67 \%$ dos casos de primavera ocorreram relacionados com o JBN reforçando mais ainda sua conexão com os SCMs. O JBN está fortemente relacionado com a convecção organizada no sul da AMS, Saulo et al. (2007) realizaram experimentos usando modelos regionais que demonstraram que o jato de baixos níveis gera convergência de umidade e calor dando início a convecção e a liberação de calor latente o que reforça a convergência durante o desenvolvimento do SCM dando assim um maior tempo de vida para o sistema. A liberação de calor latente é uma importante fonte de energia para o desenvolvimento de um SCM, em um estudo prévio Rocha (1992) utilizou simulações numéricas para observar o papel da liberação de calor latente no crescimento de um SCM e os resultados determinaram que esse é um processo fundamental para evolução dos SCM e sem ele o sistema decai rapidamente.

Para a simulação dos eventos foram selecionados dois diferente tipos de SCMs, um linear e o outro circular, o segundo é também chamado de complexo convectivo de mesoescala (CCM), de acordo com os critérios estabelecidos por Maddox (1980) um SCM será considerado um CCM quando a excentricidade do sistema convectivo for $>0.7 \mathrm{e}$ a temperatura de brilho for inferior à $-52^{\circ} \mathrm{C} \mathrm{em}$ pelo menos $50.000 \mathrm{~km}^{2}$ no interior do SCM. Portanto, SCMs com excentricidade $<0.7$ são considerados lineares.

Maddox (1983) usou dados de radiossondagem para analisar 10 eventos de CCM ocorridos na América do Norte (AMN) a fim de encontrar as características sinóticas típicas que governavam o ambiente de larga escala durante esses eventos e o que se observou foi que esses complexos convectivos se formam em ambientes com características mais barotrópicos e condicionalmente instáveis com forte advecção quente e úmida em baixos níveis em uma região frontal quase estacionária interagindo com um fraco cavado de onda curta em níveis médios. Anabor $(2008)$ realizou um estudo semelhante para SCMs na AMS, e os resultados indicam que CCMs se formam em regiões com características físicas e padrões sinóticos semelhantes aos documentados por Maddox para AMN. Já os SCMs lineares, diferente dos CCMs, se formam em regiões mais baroclínicas próximas a frentes frias onde o sistema de propaga com o avanço do jato de altos níveis (JAN). Nesses casos o avanço da frente (e do JAN) contribuem para organizar a convecção de forma linear o que caracteriza esse tipo de SCM. Os dois diferentes eventos estudados nesse trabalho foram selecionados de acordo com os padrões sinóticos típicos documentados por Maddox (1983) e Anabor (2008).

A importância de estudar esses sistemas convectivos que ocorrem nas regiões sul e sudeste da AMS durante as estações quentes é enfatizado em um estudo de (Nesbitt et al. 2006), onde o autor afirma que quase $90 \%$ do total de precipitação na bacia do rio Prata é contribuição de SCMs, este é um resultado que demonstra a importância de estudar esses fenômenos, pois eles são determinantes para o ciclo hidrológico na bacia do Prata e para a manutenção do clima local.

A forma de análise dos eventos abordada nesse trabalho foi inspirada em um estudo de Hart, R.E. (2003) sobre o ciclo de vida dos ciclones a partir do uso de parâmetros envolvendo o vento térmico e a espessura no interior dos ciclones (em duas diferentes camadas 900-600hpa e 600-300hpa) usando diagramas de fases. A análise feita por Hart para um ciclone foi adaptada nesse trabalho para um SCM, porém, novos parâmetros envolvendo o cisalhamento direcional do vento e a inclinação da espessura foram utilizadas com o objetivo de descrever a evolução do SCM com base nesses parâmetros. Nesse trabalho será analisado como o modelo WRF simula os diferentes eventos em termos desses dois parâmetros desde a iniciação até a dissipação de ambos os sistemas convectivos.

\section{Materiais e métodos}

\subsection{Identificação dos eventos}

Um método de rastreamento automático de SCMs usando imagens do satélite GOES permitiu a identificação de uma grande quantidade de eventos, de maneira que, para cada imagem de satélite processada calculava-se diversos parâmetros relativos ao SCM como área, excentricidade, coordenadas do centro geométrico entre outros. A informação sobre a excentricidade e a área dos sistemas permitiu distinguir os sistemas circulares dos 
lineares considerando apenas o valor da excentricidade no momento da máxima área dos SCMs, e com base nessas informações e nas próprias imagens de satélite foi realizada a seleção dos dois eventos aqui estudados.

\subsection{Dados e simulação}

Para a simulação dos SCMs com o modelo WRF foram usados os dados de reanálise do CFSR com resolução espacial de $0.5^{\circ}$ e resolução temporal de $6 \mathrm{~h}$. Como os horários dos dados e os horários das imagens de satélite não coincidiram perfeitamente foi necessário um arredondamento que aproxima a hora das imagens de satélite correspondentes a iniciação e dissipação dos SCMs para a hora mais próxima a dos dados do CFSR, com o propósito de selecionar o intervalo de ocorrência de cada evento e usar os dados respectivos a esse intervalo para fazer a simulação. Ambos os eventos tiveram duração de $12 \mathrm{~h}$, o SCM linear ocorreu entre as 00UTC e 12UTC do dia 30 de Novembro de 2009 e o CCM ocorreu entre as 06UTC e 18UTC do dia 18 de Novembro de 2009. A simulação no WRF foi feita com resolução espacial de $14 \mathrm{~km}$ e resolução temporal de $1 \mathrm{~h}$ para cada evento, e com os resultados foi possível associar os dados horários da simulação com a informação das coordenadas centrais dos SCMs fornecidas para cada imagem de satélite pelo método de rastreamento automático. Contudo, foi possível calcular as variáveis de interesse no centro dos SCMs em intervalos horários durante as $12 \mathrm{~h}$ de ocorrência dos sistemas.

Foram utilizados dois parâmetros para descrever a evolução dos SCMs, a inclinação da espessura (B) e o cisalhamento direcional do vento (CDV).

\subsection{Parâmetros}

A inclinação de espessura é calculada a partir do gradiente de espessura (1000-500hpa), de forma que subtrai-se o valor da espessura três graus a norte do centro do SCM pelo valor da espessura três graus a sul do SCM de acordo com a equação a baixo.

$$
B=e s p_{n}-e s p_{s}
$$

Onde B é a inclinação da espessura, espn é o valor da espessura três graus para o norte do centro do sistema e esps é o valor da espessura três graus para sul do centro do sistema. Essa diferença indica a intensidade do gradiente de espessura no sentido N-S no interior do sistema, onde valores altos e positivos de B indicam que a região onde está o SCM tem um forte gradiente de espessura, ou seja, a espessura aumenta rapidamente para o norte) e portanto corresponde a uma atmosfera com forte baroclinia e valores de $\mathrm{B}$ pequenos ou próximos de zero indicam uma atmosfera com pouca baroclinia (barotrópico), ou seja, o SCM se encontra em uma região com fraco gradiente de espessura. Valores negativos de B indicam que a espessura aumenta para o sul o que ocorre apenas no hemisfério norte, portanto nos eventos estudados nesse trabalho não haverão valores negativos de $\mathrm{B}$.

A importância da abordagem desse parâmetro deriva dos estudos realizados por Maddox (1983) e Anabor (2008), pois se CCMs tendem a desenvolver-se em uma atmosfera com características mais barotrópicas e SCMs em uma atmosfera com características mais baroclínicas, então a informação sobre o gradiente de espessura nas regiões onde ocorrem os sistemas convectivos pode ser determinante para a análise do ciclo de vida dos SCMs. A figura 1 apresenta uma configuração aleatória para ilustrar como que o parâmetro B foi calculado.



Figura 1 - Ilustra como foi calculado o parâmetro B. Sendo que a região hachurada da figura é a espessura e os contornos são de pressão a nível médio do mar

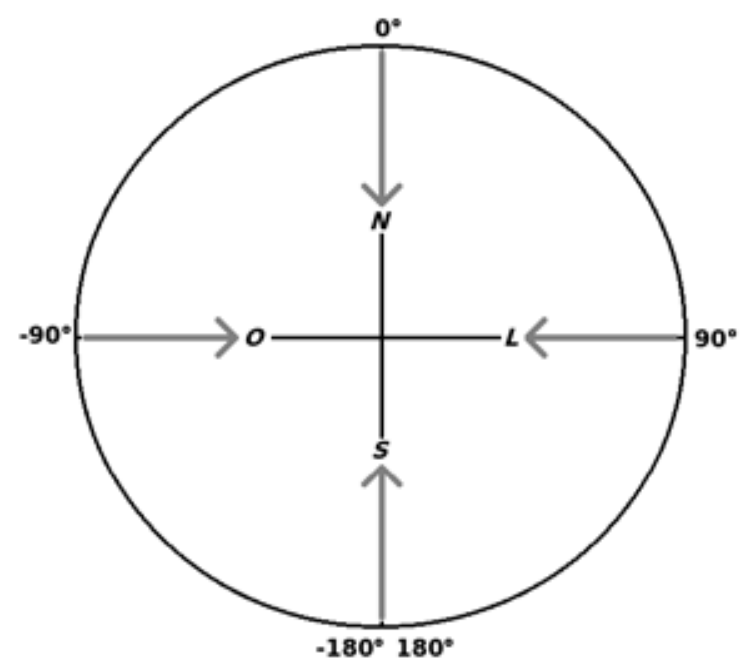

Figura 2 - Como é representado o ângulo meteorológico do vento segundo a equação (2). 
Outro parâmetro utilizado para a análise dos SCMs é o cisalhamento direcional do vento (CDV) que é calculado a partir da direção do vento em dois níveis. A direção do vento foi calculada de acordo com a equação (2).

$$
\varphi_{m e t}(\operatorname{deg})=\frac{180}{\pi} \times \arctan (-u,-v)
$$

Onde usa-se apenas as componentes u e v do vento para obter o valor do ângulo meteorológico correspondente a direção do vento cujo os valores variam entre $-180^{\circ}$ e $180^{\circ}$ como ilustrado na figura 2.

Onde $0^{\circ}$ representa vento de norte, $90^{\circ}$ vento de leste, $180^{\circ}$ ou $-180^{\circ}$ vento de sul e $-90^{\circ}$ representa vento de oeste. O CDV é calculado através da diferença entre a direção do vento em um nível superior e a direção do vento em um nível inferior, como demonstrado na equação (3).

$$
C D V=\phi_{\text {Nivel superior }}-\phi_{\text {Nivel inferior }}
$$

Portanto, se o valor do cisalhamento direcional do vento for igual a $0^{\circ}$ significa que a direção do vento não varia com a altura, logo o CDV é mínimo e se for igual a $180^{\circ}$ ou $-180^{\circ}$ então as direções dos vetores vento nos dois níveis serão opostas e o CDV será máximo nesse caso. Considerando que em latitudes médias a direção do vento em níveis superiores é predominantemente de oeste então valores de $\mathrm{CDV}$ entre $0^{\circ} \mathrm{e}-180^{\circ}$ representam advecção quente em baixos níveis sendo que $-90^{\circ}$ é a configuração que favorece a máxima advecção quente, pois representa vento de norte em baixos níveis e se a advecção quente é maior no nível inferior a camada se instabiliza. Valores menores que $-180^{\circ}$ ou maiores que $0^{\circ}$ representam advecção fria em baixos níveis sendo que a máxima advecção fria ocorre com CDV igual a $90^{\circ}$ ou $-270^{\circ}$ pois indica vento de sul em baixos níveis e se a advecção fria é maior no nível inferior a camada se torna estável. Esse parâmetro expressa a informação do quanto a camada entre os dois níveis está se instabilizando ou estabilizando devido a configuração dos ventos.

Os dois parâmetros foram plotados um contra o outro na forma de gráficos de fase onde os pontos plotados no gráfico representam os valores das duas variáveis para cada uma das $12 \mathrm{~h}$ de duração dos SCMs, permitindo assim, a análise de como se modificam esses dois parâmetros ao longo de todo ciclo de vida dos dois sistemas simulados com o WRF.

\section{Resultados}

O SCM que ocorreu em 30 de Novembro de 2009 apresentou um típico escudo de nuvens linear que estendeu-se desde o noroeste da Argentina até o leste do Rio grade do Sul, como pode ser visto na figura (3).



Figura 3 - Imagem do satélite GOES realçada no canal infravermelho no momento em que o SCM atingiu sua maior área.

Já o CCM que ocorreu sobre o RS e o Uruguai em 18 de Novembro de 2009, diferente do SCM, apresentou um escudo de nuvens circular, como mostra a imagem de satélite da figura 4 .

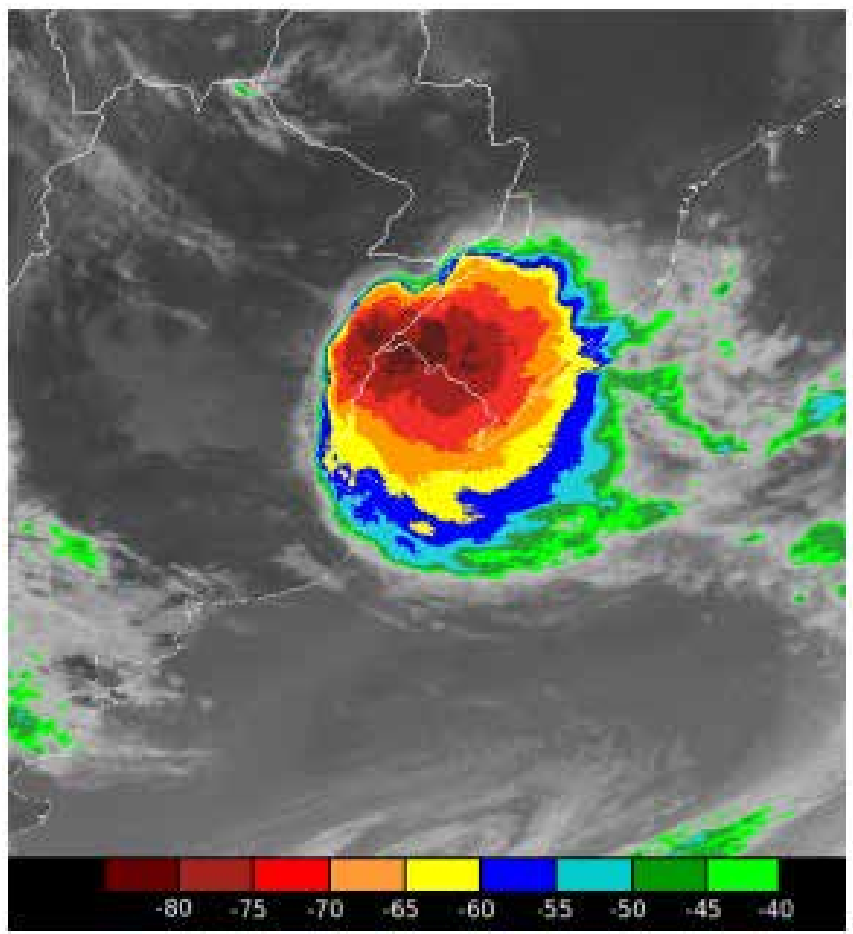

Figura 4 - Imagem do satélite GOES realçada no canal infravermelho no momento em que o CCM atingiu sua maior área. 
De acordo com os estudos realizados por Maddox (1983) e Anabor (2008) existe uma configuração sinótica típica favorável a formação de cada um dos sistemas e por isso é importante analisar o ambiente sinótico nos quais os sistemas se formaram antes de realizar a simulação.

\subsection{Análise sinótica}

Foram gerados quatro campos meteorológicos para a análise sinótica de cada evento no momento de máxima área usando os dados de reanálise do CFSR. O primeiro campo apresenta a corrente de jato em 200hpa e omega em 500hpa como pode ser visto na figura 5(a) para o caso linear e na $5(\mathrm{~b})$ para o caso circular e da mesma forma também estão apresentados os campos de pressão a nível médio do mar e espessura entre 1000-500hpa (figura 6), geopotencial e vorticidade em $500 \mathrm{hpa}$ (figura 7), linhas de corrente em 850hpa e umidade específica integrada da superfície até o nível de 200hpa (figura 8).

Em todos os campos gerados a posição do centro geométrico do SCM está indicada por um círculo com um triângulo no seu interior.

A figura 5 mostra que no momento de máxima área

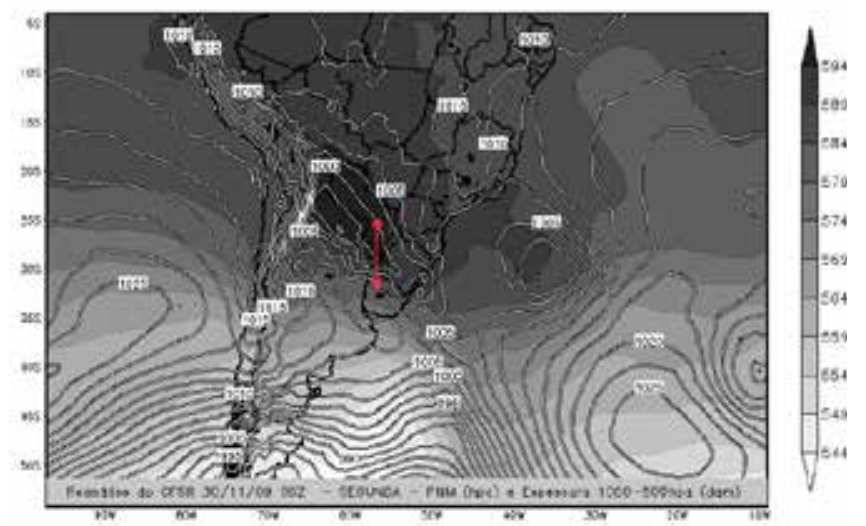

(A)

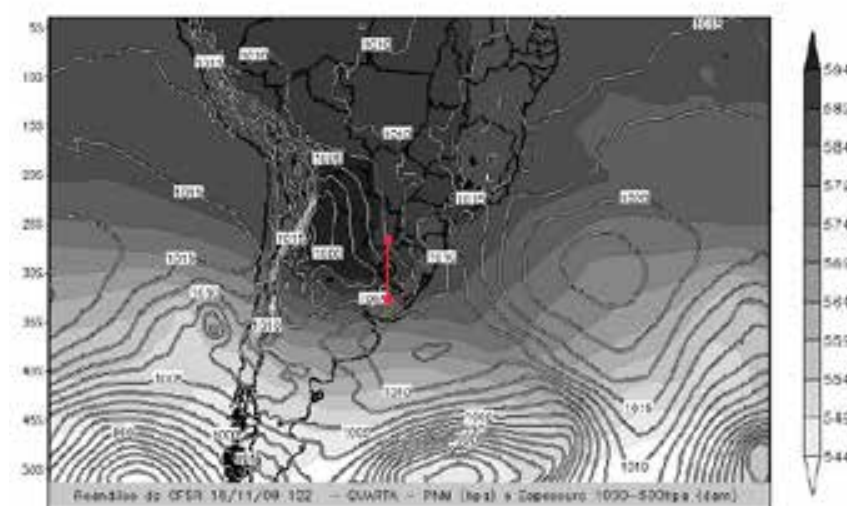

Figura 5: Pressão a nível médio do mar (contornos) e espessura entre $1000 \mathrm{hpa}-500 \mathrm{hpa}$ (hachurado) para o caso linear (a) e para o circular (b). O símbolo vermelho representa a região onde o parâmetro $\mathrm{B}$ foi calculado (como mostrado na figura 1).

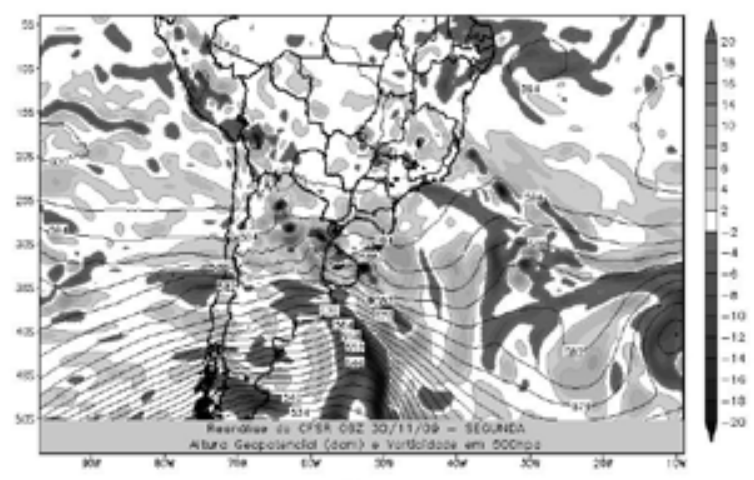

(A)

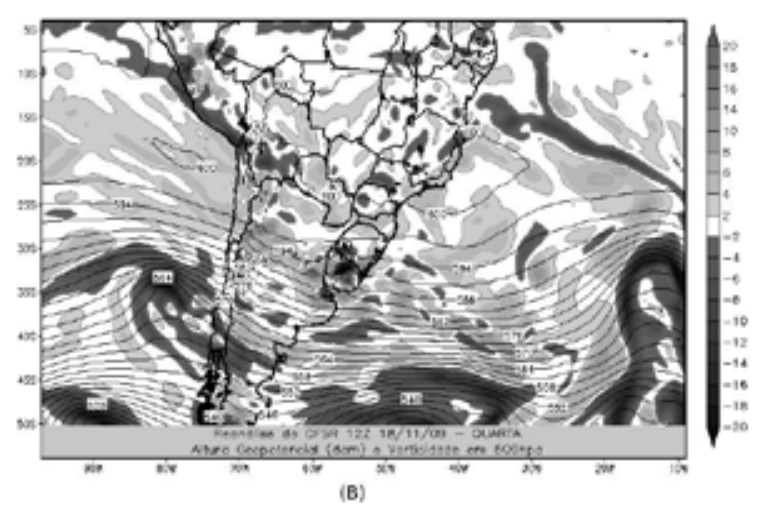

Figura 6: Geopotencial (contornos) e vorticidade (hachurado) em 500hpa para o caso linear (a) e para o caso circular (b).

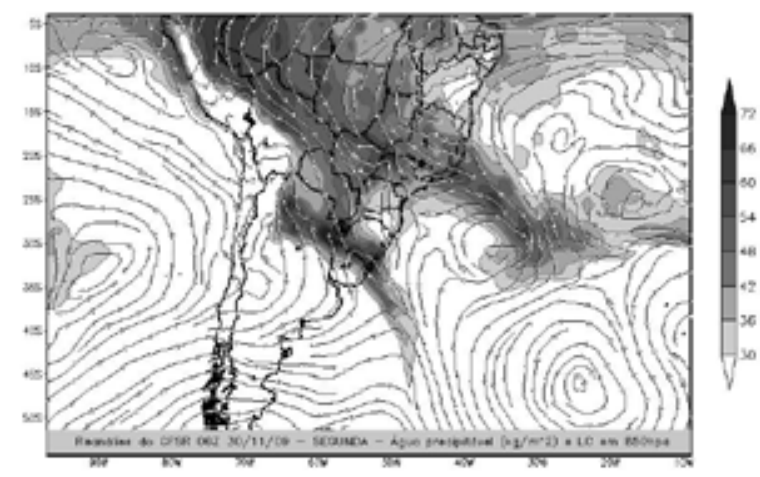

(A)

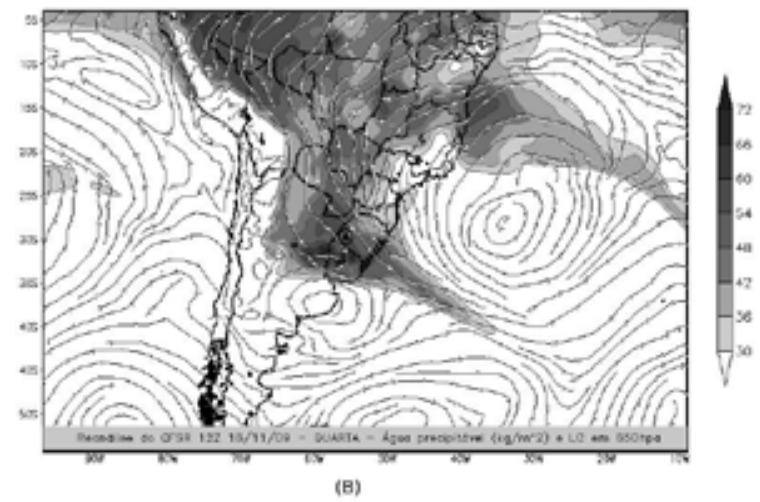

Figura 7: Linhas de corrente em 850hpa e água precipitável (hachurado) pra o caso linear (a) e para o caso circular (b). 
de ambos os eventos o jato em 200hpa se apresenta com diferentes configurações, de forma que, no caso linear, figura 5(a), percebe-se que o sistema está associado a um cavado baroclínico em altos níveis de maneira que o jato se apresenta bem inclinado com o evento ocorrendo na região de saída equatorial onde o movimento ascendente é favorecido pela circulação do jato. Nesse caso, há um acoplamento do jato polar com o jato subtropical devido ao avanço do cavado. Os contornos de omega apontam para fortes correntes ascendentes ao longo de toda região leste do cavado que está próxima da região de propagação do jato.

No CCM, figura 5(b), a magnitude do vento no núcleo do jato é um pouco maior, sua orientação é mais zonal e o sistema ocorre na região da saída equatorial relativa ao núcleo do jato subtropical, contudo, os valores de omega mostram que as correntes ascendente são menos intensas do que no SCM.

No SCM pode-se observar na figura 6(a) que existe uma forte advecção fria na região sul da Argentina associada a um ciclone extratropical que está atuando nessa região fortalecendo assim o gradiente de espessura próximo da região de avanço da frente fria, que já está bem caracterizada. O SCM ocorre dentro da zona de baixa pressão cujo centro localiza-se sobre o norte e noroeste da Argentina apresentando uma forma alongada que vai do norte do Paraguai até o litoral gaúcho, essa geometria da baixa é induzida pelo avanço do sistema frontal.

No caso circular, a zona de baixa pressão se forma sobre o norte e da Argentina e o CCM ocorre na região sudeste da baixa, mas nesse caso a zona de baixa pressão é maior do que no caso linear e estende-se para sul, pois existe um ciclone extratropical no sul do oceano atlântico que já está em oclusão, produzindo assim uma frente quase estacionária, portanto, não há avanço de sistemas frontais permitindo que a baixa do noroeste da Argentina promova um escoamento que é predominantemente de norte em toda região de ocorrência do SCM, que aliado ao fraco gradiente de espessura torna o ambiente mais barotrópico.

A figura 7(a) mostra que o SCM ocorre em uma região de advecção de vorticidade ciclônica com o avanço do cavado em 500hpa causando uma redução do geopotencial em níveis médios, que está de acordo com a redução da espessura da camada observada na figura 6(a), isso ocorre devido a baroclinia induzida pela ciclogênese extratropical que impulsiona o avanço do sistema frontal, desse modo, as isolinhas de geopotencial configuram-se na forma de uma onda com bastante amplitude.

No caso circular, figura 7(b), as isolinhas de geopotencial que estão orientadas zonalmente correspondem a uma atmosfera com pouca baroclinia e a região onde ocorre o CCM possui predominante advecção de vorticidade anticiclônica, uma vez que, a vorticidade ciclônica que aparece próxima ao sistema está sendo induzida pelo próprio CCM, portanto, o geopotencial está aumentando e a atmosfera tende a barotropia.

Na figura 8(a) é possível observar que o SCM ocorre bem próximo ao ponto de colo que está associado a ciclogênese que gerou o sistema e é uma região de forte convergência e advecção úmida em baixos níveis, que vai sendo carregada para o norte conforme o avanço da massa de ar seco que já ocupara toda a região sul e central da Argentina.

Devido a influência da frente quase estacionária no caso circular, a região de maior convergência em 850hpa se encontra mais distante do CCM, permitindo uma maior advecção quente e úmida que atua em uma área bem mais ampla do que foi observado no SCM, porém, no caso linear a umidade fica mais concentrada na região pós frontal e no caso circular a umidade espalha-se por toda região onde há advecção quente.

\subsection{Análise da simulação}

A partir dos parâmetros adotados para a análise do ciclo de vida dos SCMs foram criados dois gráficos de fases, um deles plota a inclinação de espessura (B) contra o cisalhamento direcional total (CDT), que é a soma do CDV na camada superior com o CDV na camada

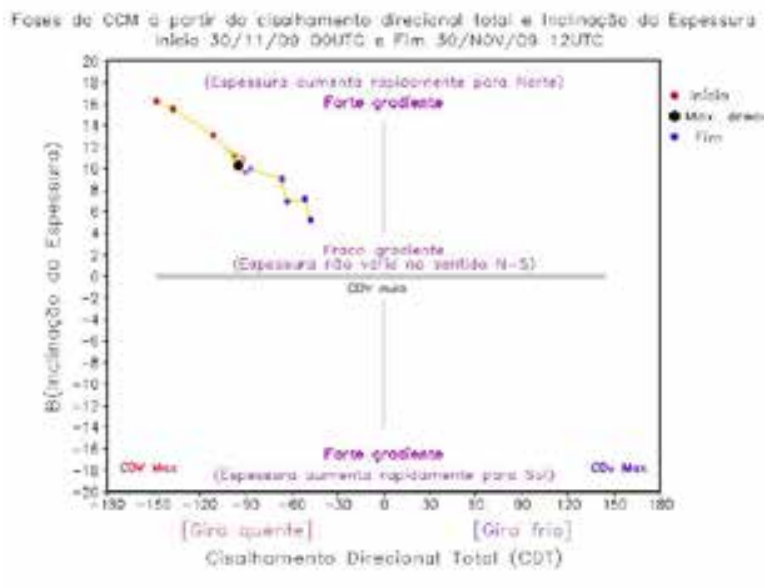

(a)

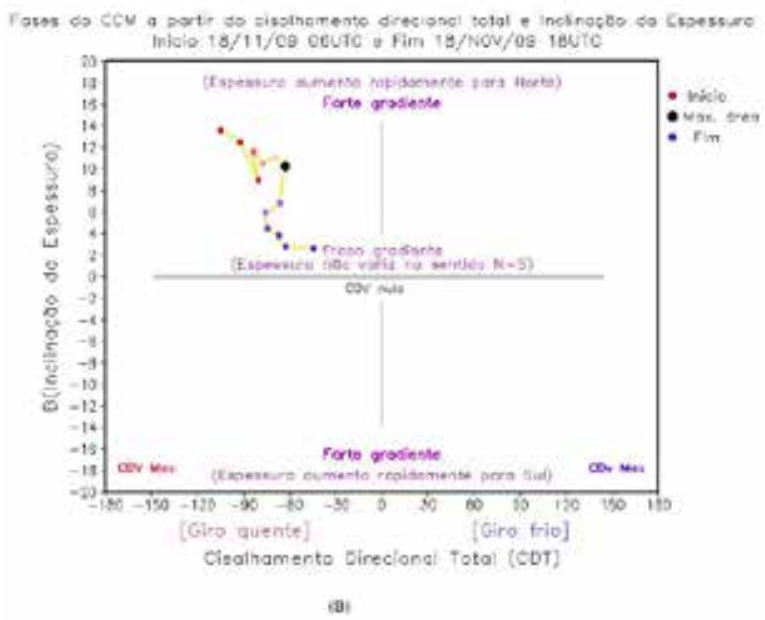

Figura 8: Inclinação da espessura vs cisalhamento direcional total para o caso linear (A) e para o circular (B). 


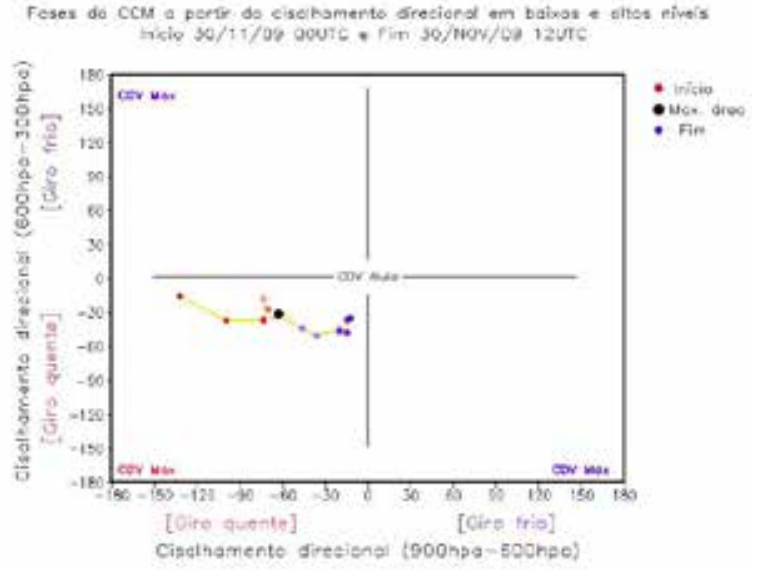

(A)

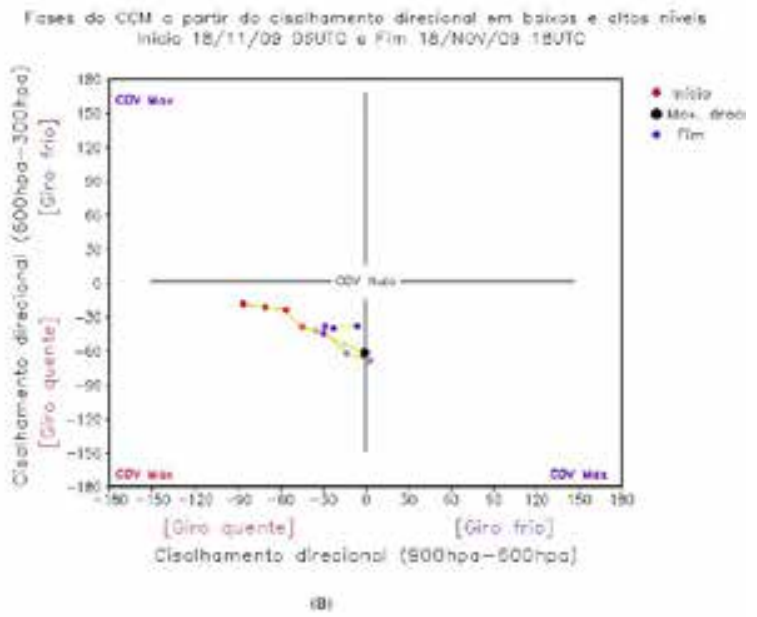

Figura 9: CDV na camada inferior vs CDV na camada superior para o caso linear (A) e para o caso circular (B)

inferior, e informa o quanto instável ou estável está toda a camada. O outro gráfico plota o CDV na camada inferior vs o CDV na camada superior permitindo assim analisar como se da a instabilização de cada camada. Os gráficos a seguir (figura 9) mostram B x CDT para os dois eventos.

Pode-se constatar a partir dos gráficos de fase da figura 9 que ambos os sistemas ocorrem em regiões de advecção quente em baixos níveis, uma vez que, os valores de CDT são sempre negativos, mas no caso do SCM o sistema inicia-se em um ambiente mais instável e com maior gradiente de espessura e conforme os sistemas crescem o ambiente vai perdendo baroclinía em ambos os casos, entretanto, essa taxa de redução é maior no caso circular, pois após seis horas (máxima área de ambos sistemas) os dois SCMs possuem valores de B praticamente iguais (aproximadamente 10), a diferença está no CDV que é maior no caso linear. Após os sistemas atingirem a máxima área o CCM continua apresentando uma queda rápida no gradiente de espessura indicando que o ambiente está tendendo fortemente a barotropia, entretanto, o CDT aumenta ligeiramente instantes após a dissipação do sistema sinalizando que a atmosfera continua instável, enquanto no SCM a queda maior é no CDT, ou seja, o sistema perde instabilidade rapidamente mas o gradiente de espessura reduz lentamente até a dissipação do SCM. Portanto, no caso linear o CDT tem uma variação maior, o que significa dizer que a configuração dos ventos sofre maiores alterações nas duas camadas durante a ocorrência do SCM, e o gradiente de espessura reduz lentamente, opostamente a isso, no caso circular o CDT tem uma variação menor e o gradiente de espessura reduz rapidamente no interior do CCM.

O outro gráfico de fase gerado para a análise da simulação plota o CDV em uma camada superior (600-300hpa) contra o CDV em uma camada inferior (900-600hpa) e está apresentado na figura a seguir para os dois eventos.

No caso linear, figura 10(a), o sistema se forma em um ambiente com CDV grande na camada inferior e vai reduzindo gradualmente ao longo de toda duração do sistema até sua dissipação, já na camada superior o $\mathrm{CDV}$ começa pequeno e adquire valores maiores próximo ao momento de máxima área e ao atingir o estágio de dissipação o CDV volta a diminuir na camada superior.

Já a figura 10(b) mostra que no momento da iniciação do CCM o CDV na camada inferior é bem menor do que no caso linear e reduz mais rapidamente, de maneira que, ao atingir a área máxima o CDV é praticamente zero, em seguida, o CDV aumenta momentaneamente e só retorna para valores próximos de zero quando o sistema já está quase dissipando-se. Na camada superior o CDV é pequeno no momento inicial, mas aumenta significantemente até atingir máxima área possuindo um valor bem maior do que o observado no SCM e instantes após isso o CDV volta a diminuir na camada superior.

Os dois eventos se formam em ambientes instáveis, entretanto, o caso linear começa mais instável e o caso circular apresenta uma pequena instabilização alguns momentos antes da dissipação do sistema o que não ocorre no SCM. Na camada superior ambos os eventos apresentam uma maior instabilização próximo ao momento de maior área, que pode estar associado a divergência em altos níveis causada pela própria tempestade, visto que, o sistema encontra-se em um estágio maduro.

\section{Conclusões}

A simulação dos sistemas convectivos usando o modelo WRF demonstrou que, com relação ao gradiente de espessura o caso linear apresentou um forte gradiente no momento inicial que foi reduzindo quase linearmente ao longo de toda duração do SCM atingindo um valor de $B$ próximo de cinco no instante que o sistema se dissipa, diferente do caso circular, que tem início em um ambiente com menor gradiente de espessura sofrendo uma queda abrupta após o momento de máxima área, até que o CCM se dissipe em uma atmosfera com fraco gradiente de espessura, onde B é próximo de dois.

$\mathrm{Na}$ camada inferior do SCM o giro do vento é maior, 
ou seja, o CDV é maior no momento inicial e portanto o ambiente é muito instável, mas com o tempo o sistema vai perdendo instabilidade rapidamente e ao final do evento o CDV na camada inferior é muito pequeno sinalizando que a direção do vento não varia com a altura.

$\mathrm{O}$ caso circular se forma em um ambiente pouco menos instável do que no caso linear, sendo que, o CDV reduz rapidamente até atingir valores próximos de zero no momento de máxima área, o que indica uma configuração estável. Logo após a fase de maturação do CCM o CDV aponta uma momentânea instabilização da camada inferior que pode ter sido estimulada pela influência da própria tempestade através da produção de uma frente de rajada que torna o CCM instável novamente após sua maturação possibilitando a formação de novas células convectivas.

A análise do CDV na camada superior indicou que os dois SCMs iniciam-se com baixos valores de CDV, sendo que, a maior instabilização ocorre próximo ao momento em que ambos os sistemas atingem área máxima, isso pode ter ocorrido pois a medida em que os sistemas vão crescendo e se tornando mais profundos é possível que ocorram perturbações no escoamento em níveis superiores, portanto, a camada torna-se mais instável no estágio de maturação. Quando o sistema entra em processo de dissipação o cisalhamento direcional volta a diminuir indicando que a camada superior está tornando-se estável novamente.

\section{Agradecimentos}

À FIPE (Fundo de incentivo a Pesquisa) pela concessão de bolsa ao primeiro autor.

\section{Referências}

Anabor, V., 2004: Descriptive analyses of meso- $\alpha$ convective systems by GOES-8 satellite images. M.S. thesis, Departament of Remote Sensing, Universidade Federal do Rio Grande do Sul, 78 pp.

Hart, R.E., 2003: A cyclone phase space derived from thermal wind and thermal asymmetry. Mon. Wea. Rev., 131, 585-616.

Maddox, R. A., 1980: Mesoscale convective complexes. Bull. Amer.Meteor. Soc., 61,1374-1387.

Stensrud, David J.;de Moraes, Osvaldo L. L.;ANABOR, V., 2008: Serial Upstream-Propagating Mesoscale Convective System Events over Southeastern South America. Monthly Weather Review .
Marengo, J. A., W. R. Soares, C. Saulo, e M. Nicolini, 2004: Climatology of the low-level jet east of the Andes as a derived from the NCEP-NCAR reanalyses: Characteristics and temporal variability. J. Climate, 17, 2261-2280.

Rocha, R. P., 1992: Numerical simulation of a mesoscale convectvie system over South America. M.S. thesis, Institute of Astronomy, Geophysics and Atmosferic Science of USP.

Nesbitt, S.W., R. Cifelli, e S.A. Rutledge, 2006: Storm Morphology and Rainfall Characteristics of TRMM Precipitation Features. Mon. Wea. Rev., 134, 2702-2721. 